\title{
MITTEILUNGEN
}

\section{Cabinet Recruitment and Parliamentary Careers. Eine internationale Tagung des Sonderforschungsbereichs 580 im Alten Schloss Dornburg am 7./8. November 2008}

Schon seit Walter Bagehots scharfsinniger Analyse der „nearly complete fusion of the executive and the legislative powers" gilt eine auch in personeller Hinsicht enge Verknüpfung von Kabinett und Parlament als wichtiges Merkmal parlamentarischer Demokratien. Dennoch fehlt es bislang an einem allgemein konsensfähigen analytischen Ansatz zur Erforschung der Logiken von Karrieren zwischen Mandat und Ministeramt. Auch die empirischen Befunde zu den entsprechenden personellen Verknüpfungen wie zur Rekrutierung der Kabinette insgesamt sind in aller Regel räumlich und zeitlich begrenzt.

Insoweit betrat das Teilprojekt A3 des Sonderforschungsbereichs „Gesellschaftliche Entwicklungen nach dem Systemumbruch“ der Universitäten Jena und Halle ein Stück Neuland, als es zur Tagung „Kabinettsrekrutierung und parlamentarische Karrieren“ in das Alte Schloss Dornburg einlud. Zu den zentralen Anliegen der Tagung gehörte die Diskussion neuerer Befunde zur Funktion der Parlamente bei der Kabinettsbildung, zu Positionssequenzen bei ministeriellen Karrieren sowie zu den Folgen institutioneller Veränderungen auf die Rekrutierung von Ministern. Gerade vor dem Hintergrund eines Bedeutungswandels der Parlamente sollte dabei das Verhältnis zwischen Parlament und Regierung auch in Hinsicht auf ihre personelle Verknüpfung einer Neubewertung unterzogen werden, wie Michael Edinger (Friedrich-Schiller-Universität Jena) zu Beginn der Tagung stellvertretend für die Organisatoren ausführte.

Die „Tagung zum Ministerköpfejagen“, wie die Frankfurter Allgemeine Zeitung in journalistischer Verknappung titelte, gliederte sich in zwei Panels: Das erste - geleitet von Heinrich Best (Friedrich-Schiller-Universität Jena) - widmete sich neueren Untersuchungen zu Ministerkarrieren in Deutschland. Daran schlossen sich im zweiten - unter der Leitung von José Magone (Fachhochschule für Wirtschaft Berlin) - Beiträge aus dem europäischen Ausland und aus der international vergleichenden Forschung an. Dadurch konnte die deutsche Entwicklung in einen europäischen Kontext eingebettet werden, um neben globalen Trends auch kulturelle und institutionelle Unterschiede aufzuzeigen, die für die Stellung der Parlamente bei der Regierungsbildung relevant sind.

Der Frage nach der Verknüpfung von Parlaments- und Kabinettskarrieren ging im ersten Panel André Kaiser (Universität zu Köln) am Beispiel der deutschen Bundeskabinette von 1949 bis 2007 nach. Kaiser ermittelte eine wachsende Bedeutung der Landesregierungen als Karrieresprungbrett für spätere Bundesminister. Zwar wurden die meisten Mitglieder der untersuchten Bundeskabinette aus den Reihen parlamentarischer Führungskräfte der Regierungsfraktionen rekrutiert, doch seit 1998 deutet sich ein Trend an, Kabinettsmitglieder zu ernennen, die bereits Ministerposten in einer Landesregierung innehatten. Kaiser begründete dies einerseits mit der durch die Regierungswechsel begründeten Suche nach kompetenten und politisch bewährten Kandidaten wie auch damit, dass die Mitglieder von 
Landesregierungen durch die föderalistische Struktur Deutschlands Erfahrungen in der Bundespolitik sammeln können. Der skizzierte Trend mindert das Bemühen der Bundesminister um eine parlamentarische Verankerung nicht. So versuchten Minister, die von einem Landes- in das Bundeskabinett wechselten, in aller Regel bei der folgenden Wahl, ein Mandat im Bundestag zu erlangen.

Lars Vogel (Friedrich-Schiller-Universität Jena) betrachtete in diesem Zusammenhang die Rolle der Landesparlamente und des Deutschen Bundestags als Rekrutierungspools für Kabinettsmitglieder. Generell scheint sich die Bedeutung einer Parlamentsmitgliedschaft als alleiniges Kriterium für die erstmalige Ministerernennung zu verringern. So stieg seit den siebziger Jahren die Anzahl der Minister ohne vorherige Parlamentserfahrung an. In den ostdeutschen Bundesländern sind für die Rekrutierung Spitzenpositionen in der Partei ebenso wichtig wie in der Fraktion. Zudem unterscheiden sich die späteren Minister bereits vor dem Eintritt ins Parlament von den übrigen Abgeordneten durch einen höheren Grad politischer Professionalisierung: Sie übernehmen mehr politische Funktionen, erlangen früher ihr Mandat und machen daher die Politik früher zu ihrem Lebensunterhalt. Obwohl Minister zunehmend von außerhalb des Parlaments ernannt werden, versuchen sie schnellstmöglich ein Mandat zu erlangen. Dies, so Vogel, zeige unter anderem, dass die Integration von Kabinett und Mehrheitsfraktion durch personelle Verschränkung weiterhin notwendig ist. Ferner werde dadurch die enge Verknüpfung von parlamentarischer und außerparlamentarischer politischer Karriere verdeutlicht, vor allem da vorrangig ein Parlamentsmandat ein Leben von der Politik ermöglicht. Die Bedeutung des Parlaments für die Ministerrekrutierung variiere also in dem Ausmaß, in dem die Anzahl beruflicher Alternativen in der Politik, die ähnliche Möglichkeiten der Professionalisierung bieten, zu- oder abnimmt.

Franz Urban Pappi (Universität Mannheim) stellte eine Untersuchung relevanter Einflussfaktoren auf Kabinettsumbildungen nach Wahlen vor. Im Mittelpunkt standen hierbei die Entlassungen von Ministern nach Stimmenverlusten der regierenden Parteien. Als theoretische Erklärungsmodelle stellte Pappi die Principal-Agent-Theorie und Gamsons Gesetz zur Ressortverteilung in Koalitionsregierungen gegenüber. Seine Analyse deutscher Landesregierungen zeigte einen deutlichen Vorsprung an Erklärungskraft für Gamsons Gesetz. Demnach scheint die Leistungsbeurteilung eines vorangegangenen Kabinetts nicht für dessen Umstrukturierung ausschlaggebend zu sein. Vielmehr, so Pappi, ist die Verteilung von Parlamentssitzen unter den Koalitionsparteien das Kriterium mit dem größten Erklärungspotential für Kabinettsumbildungen.

Im zweiten Panel präsentierte José Magone Ergebnisse einer Untersuchung portugiesischer Minister, wobei er besonders die berufliche Herkunft der Amtsinhaber hervorhob. Danach haben sich die Hochschulen zu einem bedeutenden Rekrutierungspool für Kabinettsmitglieder entwickelt. Der weitaus größte Anteil portugiesischer Minister besteht aus Universitätsprofessoren, die nach dem Ausscheiden aus dem Kabinett in aller Regel wieder ihrem Beruf nachgehen. Generell, so Magone, kommt dem portugiesischen Parlament als Sprungbrett in ein Ministeramt keine große Bedeutung zu. Stattdessen scheint bei der Kabinettsbildung die Unabhängigkeit der Regierung vom Parlament im Vordergrund zu stehen; so werden neben Wissenschaftlern vor allem Führungskräfte aus der Privatwirtschaft zu Ministern ernannt. Mit diesen Rekrutierungsmustern und der Tatsache, dass die politische Erfahrung bei der Ernennung von Amtsinhabern eine geringe Rolle spielt, nimmt Portugal nicht nur im südeuropäischen Vergleich eine Sonderrolle ein. 
Ein anderes Bild ergab Laurențiu Ştefans (Universität Bukarest) Untersuchung für Ministerkarrieren in Rumänien. Er konnte feststellen, dass die Rekrutierung der Minister erheblich nach der parteipolitischen Zusammensetzung der Kabinette variiert. Sozialdemokratische Regierungschefs tendieren verstärkt dazu, Ministerposten mit Kandidaten aus dem höheren Staatsdienst zu besetzen, während die liberal-konservativen Regierungen eher Aspiranten aus der Wirtschaft ins Kabinett berufen. Dennoch gleichen sich die Kabinette darin, dass ihnen ein durchgehend hoher Anteil ehemaliger Lehrer angehört. Insgesamt wird die Rekrutierung aus wirtschaftsnahen Bereichen immer wichtiger; das Parlament verliert hingegen als Rekrutierungsinstanz zunehmend an Bedeutung. Während das Parlamentsmandat in vielen westeuropäischen Staaten häufig den Ausgangspunkt für eine spätere Ministerkarriere darstellt, ergibt sich für Rumänien der gegenteilige Befund: Ihr erstes parlamentarisches Mandat treten Minister dort meist erst nach dem Ende ihrer Kabinettskarriere an. Das Parlament fungiert also vor allem als Auffangnetz, weniger als Karrieresprungbrett. Deutlich wird in Rumänien auch eine starke Verankerung in der Partei zur Voraussetzung für die Berufung in ein Ministeramt.

Die für Rumänien geltenden Befunde lassen sich in einer vergleichenden Perspektive für die meisten Staaten Osteuropas bestätigen, wie Katja Fettelschoß (Leuphana Universität Lüneburg) mit ihrer Untersuchung zur Bedeutung von Parlamentspositionen für Ministerkarrieren in den postkommunistischen EU-Mitgliedstaaten ermittelte. Fettelschoß zufolge stellt das Parlament dort nur für einen sehr geringen Teil der Minister den alleinigen Rekrutierungskanal dar. Vielmehr führt eine Kombination aus Parlamentsmandat und Erfahrungen in hohen Parteiämtern zur Qualifikation für ein Exekutivamt; Ämter auf lokaler und regionaler Ebene spielen keine nennenswerte Rolle. Erfahrungen in Parlamentspositionen sind also nur eine unter mehreren „Schlüsselqualifikationen“, die ein zukünftiger Minister vorweisen muss. Auch als „Auffangnetz“ dienen sie nur wenigen Kabinettsmitgliedern. Trotz allem nimmt die Bedeutung parlamentarischer Vorerfahrung für die Rekrutierung von Ministern kontinuierlich zu.

Eine andere Art der Vorerfahrung, nämlich die als so genannter Juniorminister, zu denen man in Deutschland die Parlamentarischen Staatssekretäre zählt, untersuchte Philip Manow (Universität Konstanz) international vergleichend auf ihre Relevanz für Ministerkarrieren. Generell, so Manow, variiert die Anzahl der Juniorminister in einem Land abhängig von der Fähigkeit, Parteidisziplin im Parlament durchzusetzen. Offensichtlich sind sie hier ein Instrument, um „Cabinet Government“ zu etablieren. Der Stellenwert von Juniorministerämtern für ministerielle Karriereverläufe gestaltet sich im internationalen Vergleich höchst unterschiedlich. In einigen Ländern wie Neuseeland oder Japan spielen sie nur eine untergeordnete Rolle bei der Besetzung des Kabinetts, während in Italien oder Frankreich die Berufung von ehemaligen Juniorministern zu ordentlichen Ministern die Regel beziehungsweise sogar der dominante Rekrutierungspfad zu sein scheint. Weiterhin konnte Manow feststellen, dass sich die Erfahrung in einer Juniorministerposition nicht nur förderlich auf eine Rekrutierung zum Minister auswirkt, sondern den Amtsinhabern zudem eine längere Verweildauer im Kabinett sichert.

Als Quintessenz der empirischen Beiträge lässt sich festhalten, dass die Parlamente in den untersuchten politischen Systemen deutlich in ihrer Bedeutung für die Rekrutierung von Ministern variieren, insbesondere zwischen den west- und osteuropäischen Staaten. Während sich in Osteuropa vielfältigere Rekrutierungsmuster erkennen lassen, scheint in den meisten westeuropäischen Systemen die Mitgliedschaft im Parlament weiterhin ein 
zentraler Karrierebestandteil von Ministern zu sein. Insgesamt kann jedoch auch hier der Trend zu vielfältigeren Wegen in das Ministeramt beobachtet werden, auch wenn die kulturellen, historischen und politischen Rahmenbedingungen in den einzelnen Staaten diesen Trend verschiedentlich brechen, abschwächen oder verstärken.

Vor diesem Hintergrund regte der konzeptionelle Beitrag von Luca Verzichelli (Universität Siena) eine bessere Integration der verschiedenen Datengrundlagen und Forschungsstränge an. Darin wurden die theoretischen und empirisch-praktischen Herausforderungen für die weitere Forschung angesprochen, die sich aufgrund der in den einzelnen Beiträgen geschilderten Entwicklungen ergeben. Die skizzierten Forschungsergebnisse deuten demnach auf strukturelle Veränderungen in den Karriereverläufen europäischer Minister hin. Die bisher fokussierten Karriereprozesse - Rekrutierung, Zirkulation und generelle Verlaufsmuster von Karrieren - werden, so Verzichelli, von diesen Veränderungen so sehr beeinflusst, dass es eines erweiterten Analyserahmens zur Untersuchung von Ministerkarrieren bedarf. Als potenzielle Einflussgrößen benannte er den Bedeutungsgewinn von Kernressorts, der eine größere Unabhängigkeit der jeweiligen Minister von ihrer Partei zur Folge hat, sowie die Europäisierung vieler Politikfelder. Sein vorgeschlagener Forschungsansatz verspricht gegenüber den herkömmlichen Perspektiven ein größeres Erklärungspotential für die Varianz von Ministerkarrieren.

Die in Dornburg diskutierten Befunde zeigen, dass das eingangs genannte Diktum von Bagehot durch verschiedene Entwicklungen herausgefordert wird. Insofern stellte die Tagung einen ersten Schritt dar, die Erforschung der personellen Verknüpfung zwischen Parlament und Regierung konzeptionell neu auszurichten. Eine diesen Ansatz weiterverfolgende Forschung verspricht zugleich Erkenntnisse über das generelle Verhältnis der beiden Verfassungsinstitutionen und damit zur Dynamik des parlamentarischen Regierungssystems.

Sebastian Dörl und André Nagel

\section{Die Gesellschaft zum Studium strukturpolitischer Fragen (Strukturgesellschaft). Veranstaltung zum 50-jährigen Bestehen am 5. März 2009}

Am 5. März 2009 feierte die „Gesellschaft zum Studium strukturpolitischer Fragen“ (kurz: Strukturgesellschaft) in den Räumen der Deutschen Parlamentarischen Gesellschaft ihr 50-jähriges Bestehen. Festredner waren der Präsident der Parlamentarischen Gesellschaft Prof. Dr. Heinz Riesenhuber und der Träger des Friedenspreises des deutschen Buchhandels von 2007 Prof. Dr. Wolf Lepenies. Er sprach zu dem Thema „Provinzielles Denken und Weltbürgertum: die amerikanisch-europäischen Beziehungen in der Ära Obama“.

Der Präsident der Strukturgesellschaft Dr. Ludolf von Wartenberg wies in seiner Laudatio darauf hin, dass die Gesellschaft in der breiten Öffentlichkeit wenig bekannt, aber bei aller Zurückhaltung im Auftreten eine im Parlament weitgehend anerkannte Institution sei, die Hilfe im politischen Entscheidungsprozess leisten könne, insbesondere in der Wirtschafts-, Finanz-, Sozial- und Gesellschaftspolitik. Die Strukturgesellschaft nimmt unter den Institutionen im Umfeld des Bundestages eine besondere und einmalige Stellung ein. Hinter dem etwas umständlichen Namen verbirgt sich die älteste und bekannteste dieser Vereini- 